Liesegang rings are rare, acellular, spherical to elongated, concentrically laminated, ring-like structures that range from 5 microns to 820 microns in diameter $[1,2]$. They are nonpolarizeable and are characterized by faint radial striations and an amorphous central core [3]. Liesegang rings are most commonly identified in areas of hemorrhage, inflammation, or necrosis, and in renal and perirenal cysts, and can be mistaken for parasites such as the giant kidney worm, Dioctophyma renale $[4,5]$. We describe the first known case of Liesegang rings diagnosed by endoscopic ultrasound-guided fine-needle aspiration.

A 63-year-old man with recently diagnosed esophageal adenocarcinoma was referred for locoregional staging by endoscopic ultrasound (EUS). Initial imaging studies, including computed tomography and positron emission tomography, showed no evidence of malignant lymphadenopathy or of distant metastatic disease. EUS imaging revealed an esophageal tumor that extended through the muscularis propria (stage T3) and also the presence of an echo-poor, round, smooth-bordered structure with a diameter of $1.2 \mathrm{~cm}$ in the left perirenal space (Figure 1). This was interpreted as a malignant-looking lymph node. The lesion was found to be nonvascular (on Doppler examination) and appeared to be noncystic; in particular, no postacoustic enhancement was noted.

EUS-guided fine-needle aspiration of the lesion produced a light-brown, cloudy, nonviscous fluid that contained debris. Diff-Quik staining of air-dried smears of the fluid aspirate demonstrated crystalline structures. After Papanicolaou staining, the lesion revealed the typical features of Liesegang rings, including double-layered outer walls with equallyspaced striations and an amorphous central nidus (Figure 2 ). In addition, no lymphocytes were detected and there was no evidence of malignancy. The patient received neoadjuvant chemoradiation ther-

\title{
Endoscopic Ultrasound Diagnosis of Liesegang Rings
}

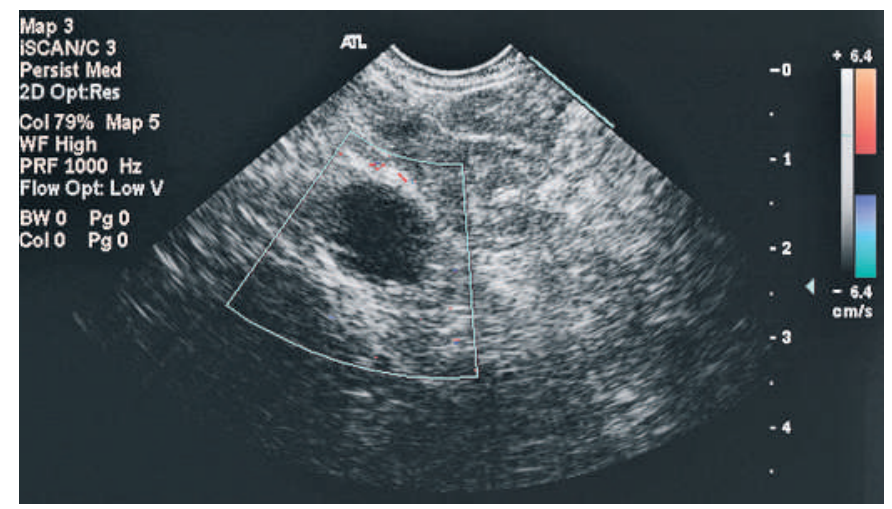

Figure 1 Endoscopic ultrasound image showing an echo-poor lesion in the left perirenal space, with no Doppler signal.

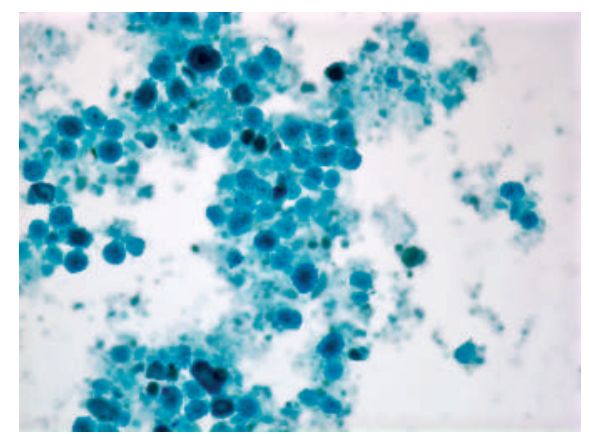

Figure 2 Liesegang rings (Papanicolaou stain, $\times 40)$.

apy, and then underwent an Ivor Lewis esophagogastrectomy. He is doing well 12 months after surgical resection of the tumor, with no evidence of recurrent disease.

Exophytic renal cysts are fairly common and easily diagnosed by EUS imaging. The presence of Liesegang rings with crystalline material and debris increases ultrasound attenuation, and this probably accounts for their noncystic appearance on ultrasound examination. While Liesegang rings have no known clinical significance, it is important that endosonographers and pathologists are aware of the phenomenon, in order to avoid misdiagnosis. This case also highlights the utility of fine-needle aspiration, which established that the lesion seen on EUS in this patient was not a perirenal lymph node, which would have designated the tumor as stage M1b and so unresectable from the curative standpoint.

\section{Competing interests: None}

Endoscopy_UCTN_Code_CCL_1AF_2AG_3AD

\section{T. E. Yusuf, M. J. Levy}

Division of Gastroenterology and Hepatology, Mayo Clinic, Rochester, Minnesota, USA.

\section{References}

${ }^{1}$ Sis B, Canda T, Harmancioglu O. Liesegang rings in a fine needle aspirate from a breast cyst. Cytopathology 2003; 14: 223-224

2 Glazier DB, Murphy DP, Cummings KB et al. Liesegang rings. J Urol 1997; 157: 940-941

${ }^{3}$ Raso DS, Greene WB, Finley JL et al. Morphology and pathogenesis of Liesegang rings in cyst aspirates: report of two cases with ancillary studies. Diagn Cytopathol 1998; 19: $116-119$

${ }^{4}$ Sneige N, Dekmezian R, Zaatari GS. Liesegang-like rings in fine needle aspirates of renal/perirenal hemorrhagic cysts. Acta Cytol 1988; 32: $547-551$

${ }^{5}$ Tuur SM, Nelson AM, Gibson DW et al. Liesegang rings in tissue: how to distinguish Liesegang rings from the giant kidney worm, Dioctophyma renale. Am J Surg Pathol 1987; 11: $598-605$

\section{Corresponding Author}

\section{T. E. Yusuf, M.D.}

Division of Gastroenterology and Hepatology, Mayo Clinic 200 First Street SW, Rochester Minnesota 55905, USA

Fax: +1-507-266-3939

E-mail: yusuf.tony@mayo.edu

Published online 3 March 2006 DOI: $10.1055 / \mathrm{s}-2006-925093$ 